\title{
Integrierte Kommunikation in der öffentlichen Verwaltung
}

Die erfolgreiche Integration der Kommunikationsaktivitäten eines Unternehmens gewinnt auch für die öffentliche Verwaltung (ÖV) an Bedeutung. Jedoch gelingt es vielen Verwaltungsbereichen noch nicht, ihr Image zu steuern. Durch eine strategische Neuorientierung und die Beachtung der Besonderheiten gegenüber der Privatwirtschaft wird interne Kommunikation zu einem erfolgreichen Marketingtool im öffentlichen Sektor.

\section{Stefanie Henkel | Kuno Schedler}

\section{Die Öffnung der öffentlichen Verwaltung}

Seit einigen Jahren sieht sich die ÖV einem höheren Anspruchsdenken ihrer Kunden gegenüber. Diese fordern mehr Kundenorientierung und besseren Service (Marson 1993; Klausegger/Scharitzer 2000). Im Vergleich zu privaten Dienstleistern hat die ÖV in diesen Bereichen großen Nachholbedarf (Bamert et al. 2004). Kern der beschriebenen Thematik ist das Image-Problem, das die ÖV trotz einiger bereits erfolgreich eingeführter Reformprojekte, noch immer hat (Finger 1997; Milakowitsch 2003). Problematisch ist vor allem, dass viele Verwaltungsstellen nicht im gewünschten Ausmaß von ihren Kunden wahrgenommen werden (Koci 2005). Es gelingt ihnen oft nicht, sich gezielt zu positionieren und den Kunden den eigentlichen Nutzen ihres Handelns zu kommunizieren. Damit ver-

„Viele Verwaltungsstellen werden nicht ausreichend oder nicht im gewünschten Ausmaß von ihren Kunden wahrgenommen."

geben sie Gelegenheiten, die Wirksamkeit ihrer Auftragserfüllung zu optimieren.

Um dieser Problematik zu begegnen, bieten sich, insbesondere vor dem Hintergrund der hohen Diversifikation an Stakeholdern, mit denen die ÖV kommunizieren muss, Maßnahmen der integrierten Kommunikation an (Bruhn 2006). Zu beachtende Spezifika sind dabei, dass nicht nur erstellte Leistungen Objekte eines integrierten Kommunikationsprozesses sind, sondern auch beabsichtigte Wirkungen, die der öffentlichen Wertschöpfung dienen (Snavely 1991). Dies kann z. B. die Öffentliche Sicherheit sein, für die Maßnahmen wie Straßensperrungen oder Polizeikontrollen notwendig sind. Zudem ist zu beachten, dass die relevanten Zielgrößen in der ÖV nicht umsatz- oder gewinnbezogen sind, 
sondern auf die Bekanntmachung und Legitimierung des öffentlichen Dienstleistungsangebotes abstellen (Redli 1984; Bruhn/Boenigk 2000; Milakowitsch 2003).

\section{Kommunikationsmanagement als Erfolgsfaktor}

Dass öffentliche Institutionen mit relevanten Anspruchsgruppen gezielt kommunizieren sollten, ist bereits früh erkannt worden (Fiorentini 1989). Bisher wurden jedoch immer nur Teilaspekte der Kommunikation im Bereich der ÖV untersucht (Pandey/ Garnett 2006). Wissenschaftliche Beiträge zur Integration und Abstimmung der verschiedenen Kommunikations-Tools im ÖV fehlen weitgehend.

Dies mag sicherlich an der noch jungen Tradition liegen, die das Thema Marketing im Öffentlichen Sektor hat. Inzwischen weisen jedoch einzelne Beiträge darauf hin, dass die Integration der Kommunikationsaktivitäten auch im öffentlichen Sektor einen Nutzenbeitrag stiften kann (Kotler 2006; Riedel 2006). Im Rahmen dieses Beitrages soll nun aufgezeigt werden, wie ein integrierter Ansatz zur Entwicklung von Kommunikationsstrategien für Öffentliche Institutionen eingesetzt werden kann. Dabei wird geklärt (1) welchen Zielerreichungsbeitrag die Integrierte Kommunikation für die ÖV leisten kann, (2) auf welche Zielgruppen diese in welchem Ausmaß abgestimmt werden muss und (3) wie eine integrierte Kommunikationsstrategie für die ÖV exemplarisch ausgestaltet werden kann.

\section{Erwünschtes Kundenverhalten im Public Marketing}

Ein Charakteristikum des öffentlichen Sektors ist, dass sich die Bürger als Steuerzahler in der Regel an der Finanzierung der meisten öffentlichen Dienstleistungen und Aktivitäten beteiligen müssen; unabhängig davon, ob sie diese Leistungen auch in Anspruch nehmen. Im Gegensatz zur Privatwirtschaft besitzt der Staat zudem die rechtliche Legitimation und Autorität, durch Vorschriften wie etwa Tempolimits für Autofahrer oder Emissionsgrenzwerte für Industriebetriebe diese quasi zu „Zwangskunden“ seiner Verwaltungstätigkeit zu machen. Vor diesem Hintergrund sieht sich die öffentliche Verwaltung zunehmend unter Druck, Art und Umfang ihrer Aufgabenerfüllung zu rechtfertigen. Die Bedürfnisse der für die ÖV relevanten Anspruchsgruppen spielen deshalb eine immer größere Rolle und werden zu entscheidungsrelevanten Größen, wenn es um die Legitimierung öffentlicher Dienstleistungen geht.

Marketing kann nun folgende Beiträge leisten: Einerseits kann es eingesetzt werden, um mit Methoden der Marktforschung die Bedürfnisse der verschiedenen Anspruchsgruppen zu analysieren, andererseits kann es helfen, deren Verhalten zielgerichtet zu beeinflussen. Während das generelle Marketingziel der „Verhaltensbeeinflussung “ auch im öffentlichen Sektor zum Tragen kommt, ergeben sich hinsichtlich des erwünschten (Kunden-)Verhaltens deutliche Unterschiede. Anders als im privaten Marketing geht es im Public Marketing nicht um Kauf, sondern um Mitwirkung,
Abb. 1 Unterschiede im erwünschten Kundenverhalten

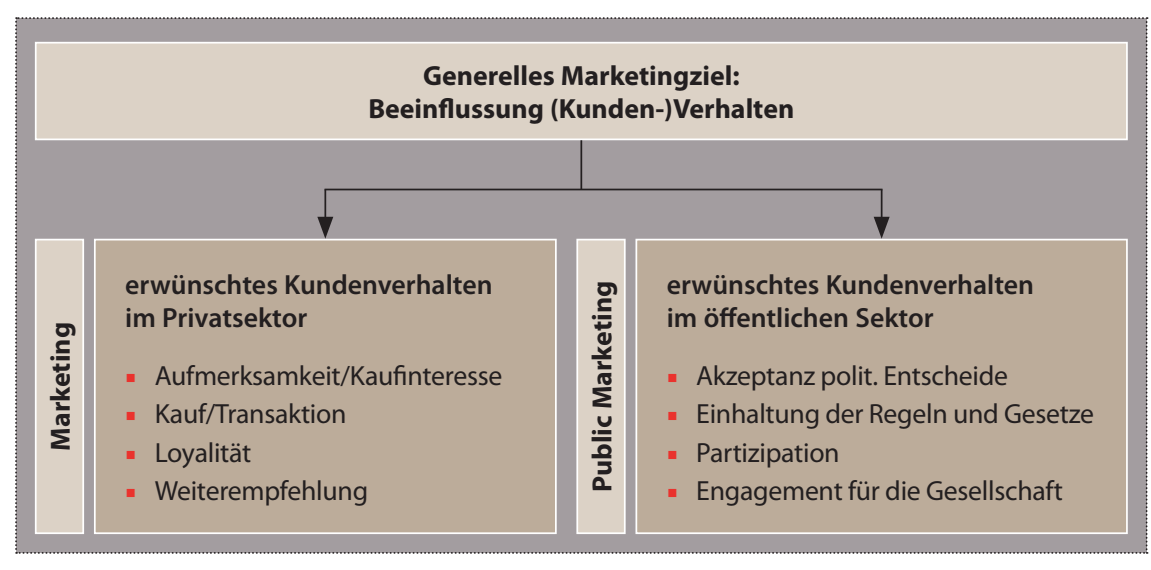

$+44$ aktive Unterstützung und Engagement (siehe Abbildung 1 und Schedler 2006).

Trotz unterschiedlicher Absichten sieht man sich jedoch im privaten sowie im öffentlichen Sektor mit ähnlichen Problemen konfrontiert. Die Kunden sind angesichts der ansteigenden Informationsflut überlastet, es wird immer schwieriger, mit Botschaften zu ihnen durchzudringen, um die nötige Akzeptanz und Aufmerksamkeit zu erlangen. Divergierende Informationen und unabgestimmte Kommunikationsaktivitäten lösen zudem Verwirrung und in der Folge eine ablehnende Haltung gegenüber öffentlichen Dienstleistungen aus.

\section{Integrierte Kommunikation als Kernprozess des Public Marketing}

Nach Bruhn (2003) ist es das Ziel der Integrierten Kommunikation durch eine intensivere Koordination der kommunikativen Aktivitäten eines Unternehmens eine effektivere und effizientere Darstellung bei den relevanten Zielgruppen zu erreichen. Zur Kommunikationszielgruppe zählt Bruhn neben den Kunden auch weitere Anspruchsgruppen des Unternehmens. Diese spielen auch bei der Entwicklung eines Konzeptes zur Integrierten Kommunikation für die öffentliche Verwaltung eine entscheidende Rolle. Im Vergleich zu privat geführten Unternehmen, verfügt die öffentliche Verwaltung über eine große Zahl von Anspruchsgruppen, die nicht nur untereinander sehr heterogen sind, sondern auch ein weitreichendes Einflusspotenzial auf die Kernprozesse der ÖV haben.

In politischen Entscheidungsprozessen versuchen die beteiligten Akteure (Politik, Bürger, Unternehmen, Medien etc.) sich durch eine bestimmte Positionierung zu profilieren. Der Einfluss dieser Akteure hängt dabei von ihrer Gestaltungsmacht ab. Als Beispiel sei hier das Bundesamt für Bevölkerungsschutz genannt, welches sowohl in der Schweiz als auch in Deutschland mit der Vorbeugung und dem Management von Katastrophen und Notfällen beauftragt ist. Befasst sich dieses Amt nun z. B. mit dem Bau eines neuen Hochsicherheitslabors, so ist im Sinne der Integrierten Kommunikation zunächst zwischen den verschiedenen Anspruchsgruppen zu differenzieren. Relevante Akteure in 
diesem Zusammenhang könnten das Bundesamt für Gesundheit, die Finanzierungskommission, Betroffene und Bürger, Verbände, Medien, sonstige politische Gremien sowie Pharmaunternehmen sein. Im Rahmen der Entwicklung einer geeigneten Kommunikationsstrategie muss das Bundesamt für Bevölkerungsschutz die genannten Akteure und ihre jeweiligen Interessen kennen und berücksichtigen.

Anhand des vorgestellten Rasters kann ermittelt werden, mit welcher Intensität das Bundesamt mit welcher Gruppe kommunizieren sollte. Dabei sollten solche Akteure, die in das obere rechte Feld der aufgezeigten Matrix einzuordnen sind, im besonderen Fokus der Kommunikationsaktivitäten stehen. Ein denkbarer Akteur im Hinblick auf das genannte Beispiel ist die Finanzierungskommission. Können die benötigten Gelder nicht bereitgestellt werden bzw. liegt keine Zustimmung zu deren Beschaffung vor, so sieht sich das Projekt einem Widerstand gegenüber, der noch zudem von einem Akteur mit einer hohen Gestaltungsmacht ausgeht. Ohne finanzielle Ressourcen lässt sich das Projekt nicht durchführen. In einem solchen Fall ist die Finanzierungskommission als „Veto-Spieler“ zu verstehen, von dem das ganze Projekt abhängt. Ist die Erfüllung einer Aufgabe hingegen von der Mitwirkung der Betroffenen abhängig (z. B. Hilfe zur Selbsthilfe im Sozialbereich), so kann man von einer hohen Gestaltungsmacht der Kunden ausgehen. Gruppen mit einer weniger hohen Gestaltungsmacht, kann mit einer geringeren Kommunikationsintensität begegnet werden. Dies könnten z.B. Verbände sein, die über wenig Einfluss im Parlament verfügen. Strategisch gesehen muss die Kommunikationsintensität umso höher sein, je widersprüchlicher die Interessenslage und je höher die Gestaltungsmacht eines Akteurs ist (siehe Abbildung 2).

Integrierte Kommunikation kann hier positive Beiträge leisten: Durch die Integration der Kommunikationsaktivitäten können Wirkungssynergien erzielt werden, die zu einer verstärkten Differenzierung und Positionierung gegenüber den Anspruchsgruppen führen. Ein einheitliches Erscheinungsbild erhöht zudem die Glaubwürdigkeit der ge-

seit 2003 in der Schweiz und seit 2004 in Deutschland troffenen Aussagen und verstärkt den Lerneffekt bei den angesprochenen Zielgruppen (Esch 2006). Durch die Integration der internen Kommunikation lässt sich darüber hinaus auch eine höhere Identifikation der Mitarbeiter mit der eigenen Institution bewirken (Bruhn 2003).

\section{Gezielte Neupositionierung öffentlicher Dienstleistungen}

Auch im Rahmen der Neupositionierung können Elemente einer integrierten Kommunikation für Öffentliche Institutionen von großem Nutzen sein. Wiederum soll hier das Beispiel des Bundesamtes für Bevölkerungsschutz herangezogen werden, da es die zu bewältigenden Problembereiche und Herausforderungen widerspiegelt, denen sich viele öffentliche Einrichtungen aktuell gegenübersehen.

Ein eigenes Amt für Bevölkerungsschutz besteht in seiner jetzigen Form, sowohl in Deutschland als auch in der Schweiz, erst seit wenigen Jahren ${ }^{1}$. $\mathrm{Zu}$ seinen primären Aufgabengebieten gehört die Erbringung von Dienstleistungen im Bereich der Katastrophenhilfe für die Länder bzw. Kantone. Wesentlich aus Sicht des Marketingstrategen ist, dass das Bundesamt im Falle von Katastro- phen nur bedingt direkt in der Öffentlichkeit in Erscheinung tritt. Die operative Bewältigung liegt bei den Ländern bzw. Kantonen und den Gemeinden. Die Wahrnehmung des direkten „Bürgernutzens“ wird folglich erst durch deren Ausführung sichtbar. Es ist deshalb nicht verwunderlich, dass seitens der Bundesämter oftmals ein Defizit des Bekanntheitsgrades festgestellt wird. Vielfach sind die erbrachten Leistungen für die Bürger aber auch für viele andere Stakeholder nicht unmittelbar erfahrbar und ein direkter Nutzen wird nicht assoziiert (Flury 2006). Ein weiterer Grund für den geringen Bekanntheitsgrad ist die erst kürzlich erfolgte Gründung bzw. Umstrukturierung. Das Schweizer Bundesamt für Bevölkerungsschutz wird z. B. immer noch als „Militärdepartement" (EMD), sein Vorsteher in erster Linie als „Verteidigungsminister" (manchmal auch als „Sportminister), nicht aber als „Bevölkerungs-“ oder „Katastrophenschutzminister" wahrgenommen. Hinzu kommt, dass die durch die Reorganisation erfolgte Zusammenführung verschiedener Dienststellen mit eigenen „Kulturen“ den Aufbau einer kohärenten Corporate Identity sowohl nach innen als auch nach außen erschwert (Flury 2006). Eine integrierte Kommunikationsstrategie, die sowohl die interne als auch die externe Kommunikation mit einbezieht,
Abb. 2 Strategisches Stakeholder Portfolio der öffentlichen Verwaltung

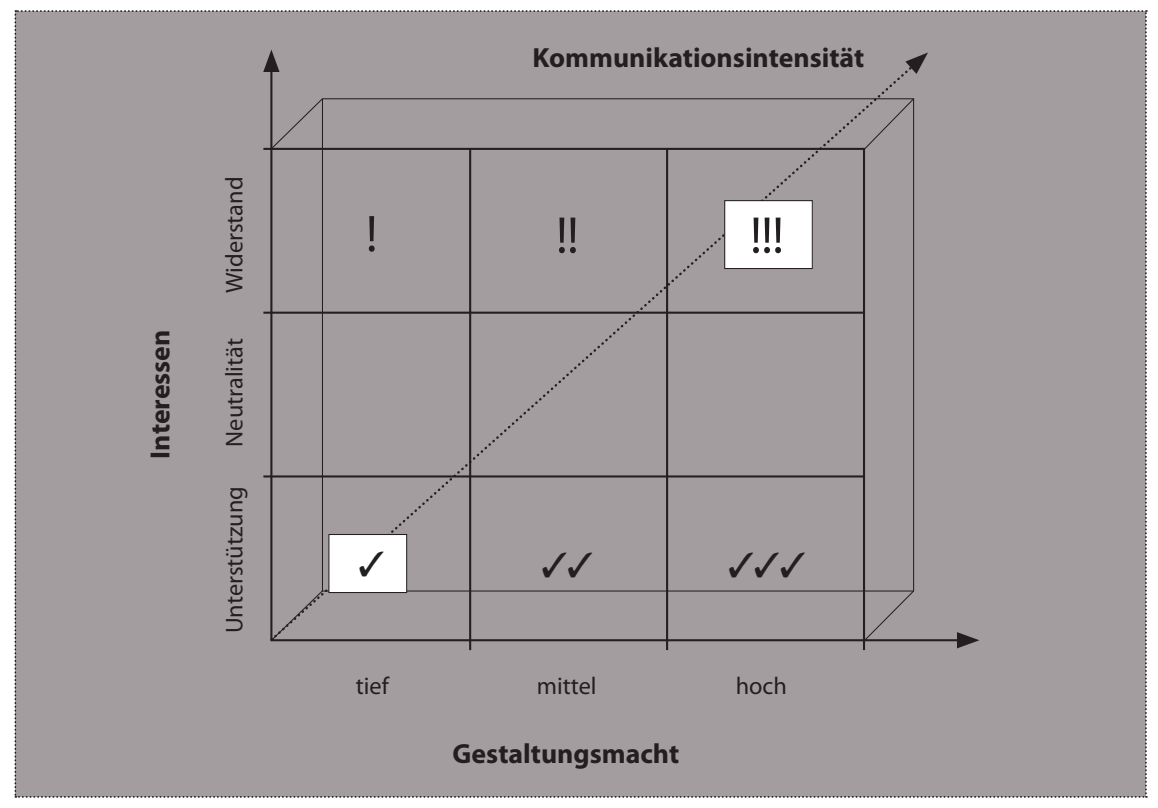


kann hier einen wertvollen Beitrag zur Erreichung spezifischer Ziele wie Neupositionierung, Steigerung der Bekanntheit bei den relevanten Stakeholdern, sowie Schaffung einer direkten Assoziation zum Aufgabenspektrum des Bundesamtes leisten. Nach Bruhn (2003) kann die Ermittlung einer Strategie zur Integrierten Kommunikation nach folgender Ordnung ablaufen (siehe Abbildung 3).

Für das Bundesamt für Bevölkerungsschutz bedeutet dies, zunächst eine Positionierungsstrategie auszuarbeiten, die die Ansprüche aller relevanten Stakeholder berücksichtigt. Die gewählte Positionierung muss dabei den Spagat zwischen politischer Kompatibilität und aktuellen Kundenbedürfnissen leisten können. Zudem sollte die Positionierung wie alle daraus abgeleiteten Komponenten ein Identifikationspotenzial für die Mitarbeiter besitzen. Der Formulierung glaubhafter und für den Mitarbeiter konkret umsetzbarer Botschaften wird auch im Marketing zunehmendes Interesse entgegengebracht. Botschaften, die von den eigenen Mitarbeitern als überzogen und nicht realisierbar wahrgenommen werden, bergen ein hohes Frustrationspotenzial und können das Zustandekommen eines einheitlichen integrierten Auftrittes behindern (Henkel et al. 2007).

Der Problematik des mangelnden Bewusstseins der verschiedenen Anspruchsgruppen hinsichtlich des zumeist nicht direkt erfahrbaren Nutzens der Leistungen des Bundesamtes für Bevölkerungsschutz, kann bspw. durch ein Leitmotiv begegnet werden. Dies kann ein Symbol, ein Slogan oder eine Figur sein, die wiederholt auftreten und mit verschiedenen Aussagen verknüpft werden kann.
Schließlich muss eine Wahl hinsichtlich der einzusetzenden Instrumente getroffen werden. Nach Bruhn (2003) ist es ratsam, zunächst ein Leitinstrument zu definieren aus dem sich, differenziert nach Zielgruppen, der weitere Instrumenteneinsatz ableiten lässt. Wie Esch (2006) in einer experimentellen Untersuchung zeigt, stiften die einzelnen Medien einen durchaus unterschiedlichen Beitrag zur Integration kommunikativer Botschaften. Unter Low-InvolvementBedingungen, zu denen ein Großteil der öffentlichen Dienstleistungen zählt, empfiehlt Esch (2006) vor allem die Integration von Schlüsselbildern.

\section{Implikationen und Fazit}

Marketing im öffentlichen Sektor verfolgt ein komplexes Zielsystem. Dies ergibt sich aus dem spezifischen Umfeld, das, geprägt durch die Interessen und Machtpositionen der einzelnen Anspruchsgruppen, eine hohe Diversifikation aufweist. Um die verschiedenen Stakeholder politisch-strategisch anzusprechen, ist ein integriertes Kommunikationskonzept nötig. Dabei ist zu beachten, dass Kommunikation in der ÖV andere funktionale Beiträge leisten muss als in der Privatwirtschaft. Folglich können Konzepte aus dem Privatsektor zwar als Vorlage für die ÖV dienen, aber nicht blind übernommen werden. Spezielle

\section{Abb. 3 Inhaltliche Ordnung eines integrierten Kommunikationskonzeptes}

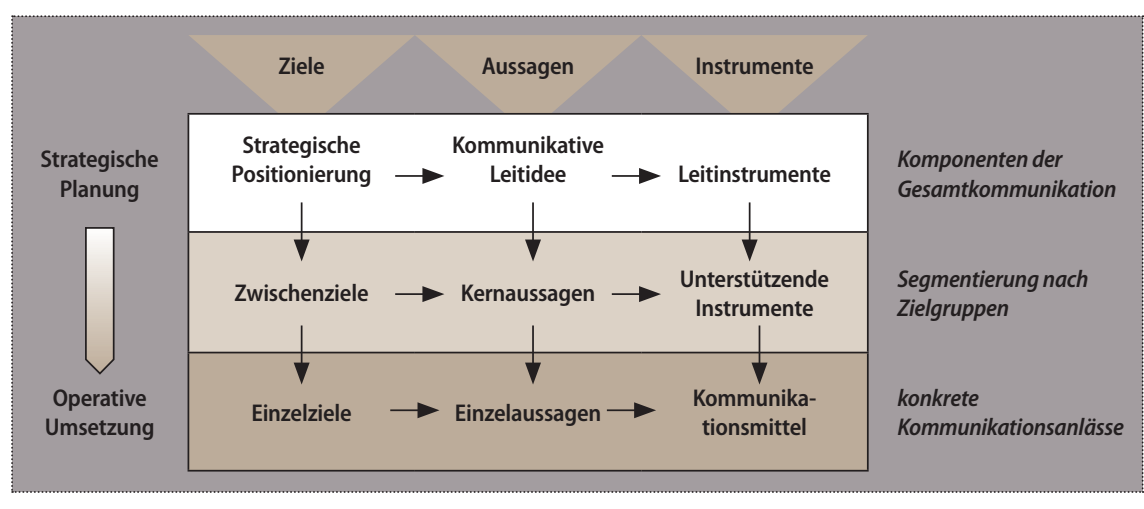

Quelle: In Anlehnung an Bruhn 2003, S. 1997
Beachtung im öffentlichen Sektor sollen daher die folgenden Punkte haben:

- Kernbotschaften sollten gegenüber allen Anspruchsgruppen, trotz divergierender Bedürfnisse und Interessen einheitlich formuliert werden.

- Die Intensität der Kommunikationsaktivitäten sollte auf die Interessenslage und die Gestaltungsmacht der jeweiligen Zielgruppe abgestimmt werden.

- Zu den Kommunikationskanälen zählen alle Kontaktpunkte, die der Kunde mit einer Botschaft haben kann. Neben klassischen Medien wie Internet oder Print ist auch die persönliche Kommunikation als wichtiger Faktor zu berücksichtigen.

Auf diese Weise kann der Ansatz zur Integrierten Kommunikation auch im öffentlichen Sektor effektiv genutzt werden und einzelne Institutionen bei der Erreichung ihrer Positionierungsziele gegenüber den relevanten Anspruchsgruppen unterstützen. Zusammenfassend ist festzustellen, dass sich hier, vor dem Hintergrund der unterschiedlichen Rahmenbedingung und Zielsysteme in der ÖV, sowohl für die Forschung als auch für die Praxis ein neuer Bereich eröffnet.

\section{„Der Formulierung glaubhafter und für den Mitarbeiter konkret umsetzbarer Botschaften wird auch im Marketing zunehmendes Interesse entgegengebracht.“}

\section{Literatur:}

Bamert, T./Wehrli, H. P./Raymann, U. (2004): Kundenzufriedenheit: Ausgewählte Branchen im Mehrjahresvergleich, Zürich.

Bruhn, M. (2003): Integrierte Unternehmens- und Markenkommunikation. Strategische Planung und Operative Umsetzung, 3. Aufl., München.

Bruhn, M. (2006): Integrierte Kommunikation, in: Schmid, B. F./Lyczek, B. (Hrsg.): Unterneh menskommunikation, Wiesbaden.

Bruhn, M./Boenigk, M. (2000): Integrierte Kommunikation in deutschen Unternehmen Ergebnisse einer empirischen Untersuchung, in: Bruhn, M./Schmidt, S./Tropp, J. (Hrsg.): Integrierte Kommunikation in Theorie und Praxis, Wiesbaden, S. 65-85.

Esch, F.-R. (2006): Wirkung integrierter Kommunikation, 4. Aufl., Wiesbaden.

Finger, M. (1997): Die Rolle des Parlaments beim New Public Management, Entflechtung, von strategischen Entscheidungen und operativer Führung, in: Der Schweizer Treuhänder, Nr. 1, S. 47-51. 
Fiorentini, G. (1989): Public Marketing in the Relationship Between Public Administration and External Environment, in: Journal of Professional Services Marketing, 5, 1, S. 47-69.

Flury, C. (2006): Public Policy Marketing: Möglichkeiten und Grenzen für die Anwendung von privatwirtschaftlichen Marketingkonzepten in der öffentlichen Verwaltung, St. Gallen.

Henkel, S./Tomczak, T./Heitmann, M./Herrmann A. (2007): Managing Brand Consistent Employee Behaviour: Relevance and Managerial Control of Behavioural Branding, in: Journal of Product and Brand Management, 14, 2, S. 1-22.

Klausegger, C./Scharitzer, D. (2000): Der Bürger als Kunde: Spezifika öffentlicher Kundenbeziehungen am Beispiel einer Analyse der Kun denzufriedenheit bei Finanzämtern., in: Jahrbuch der Absatz- und Verbrauchsforschung GfK, 46, 3, S. 280-300.

Koci, M. (2005): Servicequalität und Kundenorientierung im öffentlichen Sektor, Bern.

Kotler, P. (2006): Marketing in the Public Sector. A Roadmap for Improved Performance, Upper Sadle River, New Jersey.

Marson, D. B. (1993): Building customer-focused organizations in British Columbia, in: Public Administration Quarterly, 17, 1, S. 30-41.

Milakowitsch, M. E. (2003): Balancing Customer Service, Empowerment and Performance with Citizenship, Responsiveness and Political Accountability, in: International Public Management Review, 4, 1, S. 61-82.

Pandey, S. K./Garnett, J. L. (2006): Exploring Public Sector Communication Performance: Testing a Model and Drawaing Implications, in: Public Administration Review, 66, 1, S. 37-51.

Redli, M. (1984): Unternehmerische Führung öffentlicher Betriebe, in: Kaufman, O. K./Koller, A./Riklin, A. (Hrsg.): Zur Zukunft von Staat und Wirtschaft in der Schweiz., Zürich/ Köln, S. 197-203.

Riedel, F. (2006): Public Marketing, München/ Mering.

Schedler, K. (2006): Networked policing. Towards a public marketing approach for urban safety, in: German Policy Studies, 3, 1, S. 112-136.

Snavely, K. (1991): Marketing in the Governmen Sector, in: American Review of Public Administration, 21, 4, S. 311-325.

\section{Autoren}

\section{Dipl.-Kffr. Stefanie Henkel}

wissenschaftliche Mitarbeiterin am Institut für Öffentliche Dienstleistungen und Tourismus der Universität St. Gallen E-Mail: stefanie.henkel@unisg.ch

Prof. Dr. Kuno Schedler Professor für Betriebswirtschaftslehre mit besonderer Berücksichtigung des Public Management und Direktor am Institut für Öffentliche Dienstleistungen und Tourismus der Universität St. Gallen

E-Mail:kuno.schedler@unisg.ch
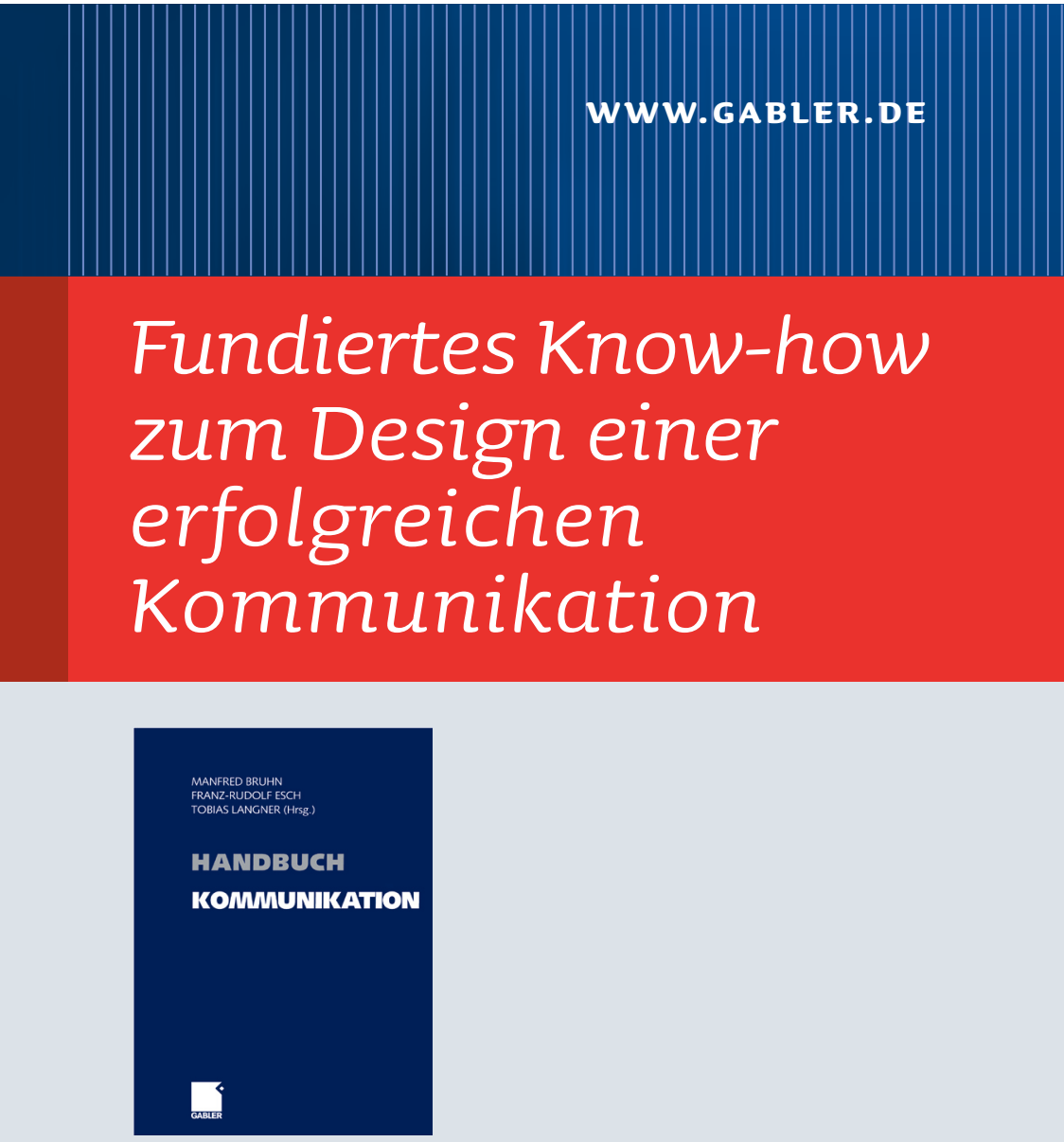

Manfred Bruhn | Franz-Rudolf Esch | Tobias Langner (Hrsg.) Handbuch Kommunikation

Grundlagen - Innovative Ansätze - Praktische Umsetzungen 2008. Ca. 1300 S. Geb. mit SU

Ca. EUR 149,00 ISBN 978-3-8349-0377-8

Das notwendige, wissenschaftlich fundierte Know-how zum Design einer erfolgreichen Kommunikation. Aufbauend auf dem verhaltenswissenschaftlichen Marketingansatz vermitteln führende Kommunikationsexperten konkrete und praxisrelevante Handlungsempfehlungen zur Konzeption, Umsetzung und Kontrolle der Kommunikation mit verschiedenen Adressaten sowie in unterschiedlichen Branchen.

Einfach bestellen:

kerstin.kuchta@gwv-fachverlage.de Telefon+49(o)611. 7878-626

KOMPETENZ IN

SACHEN WIRTSCHAFT 